\title{
OPTIMIZATION OF NON PERIODIC HOMOGENIZED MICROSTRUCTURES
}

\author{
D. CHENAIS, M.L. MASCARENHAS, AND L. TRABUCHO
}

\begin{abstract}
On considère une structure homogénéisée, qui est la limite d'un milieu formé d'un matériau élastique contenant des trous microscopiques. On cherche à ce que la distribution des trous soit telle que le matériau poreux limite satisfasse au mieux un critère donné. Nous voulons avoir accès à des milieux non périodiques. Lorsqu'il y a des trous, la méthode de la H-convergence n'est plus directement utilisable. Nous utilisons ici la modélisation par milieux quasi-périodiques introduite par Mascarenhas-Polisevski, et les techniques d'optimisation de forme. Nous donnons un procédé qui peut être utilisé numériquement pour calculer la différentielle du critère qui mesure la qualité du matériau homogéneisé.
\end{abstract}

Key words: homogenization, shape optimization, elasticity

\section{INTRODUCTION}

We consider an homogenized structure which is the limit of a given material mixed with holes. We would like the distribution of the holes to be as good as possible with respect to a given criterium measuring its behaviour.

For the case of a mixture of two elastic materials, and for the minimization of the observance, theoretical results are given by Allaire, Francfort, Kohn, Murat, Tartar, using H-convergence techniques. When there are holes this technique theoretically fails.

We present a way to handle the case of holes and different kinds of functionals to be minimized, using a quasi-periodical modelization of the material. Notice that the method we present is related to numerical methods proposed by Kikuchi and Bendsoe (cf [1]), based on an idea of Kohn and Strang (see [2], [3]). It is built by using periodical cells containing holes which change from cell to cell. For the case of the torsion of a bar, the homogenization of the material is done by Mascarenhas-Polisevski in [4]. The results are recalled in section 2.1. We present the results in a way which is adapted to the techniques of optimal design that we recall in section 3 . Then using this method, we prove that the criterium which has to be optimized is differentiable, and we give an explicit procedure to compute this differential. This can be used numerically in a descent method.

In this paper we only treat the case of the torsion of a bar, but the method can obviously be generalized to several other mechanical systems.

\section{SETting of The PRoblem}

\subsection{Modelling OF THE TORSION OF A QUASI-PERIODIC HOMOGENIZED BAR}

Let $\Omega$ be an open, bounded, connected and Lipschitz subset of $\mathbf{R}^{2}$, and $Y=] 0,1\left[^{2}\right.$. For each $x \in \Omega$, let $T(x) \subset Y$ be a closed, regular, connected 
subset of $Y$, and $Y^{*}(x)$ be the set $Y \backslash T(x)$. We consider the set:

$$
\bigcup_{x \in \Omega}\left[\{x\} \times Y^{*}(x)\right] \subset \Omega \times Y
$$

extended by periodicity to the entire $\Omega \times \mathbf{R}^{2}$, and denote by $\chi$ its characteristic function.

For each small positive parameter $\varepsilon, \mathbf{R}^{2}$ is covered by the squares $Y_{\varepsilon k}=\varepsilon Y+\varepsilon k$, where $k \in \mathbf{Z}^{2}$. Let $\mathbf{Z}_{\varepsilon}$ represent the set of all $k \in \mathbf{Z}^{2}$ such that $Y_{\varepsilon k}$ is included in $\bar{\Omega}$. We define $\Omega_{\varepsilon} \subset \Omega$ by the following characteristic function:

$$
\chi_{\varepsilon}(x)= \begin{cases}\chi(x, x / \varepsilon) & x \in Y_{\varepsilon k}, \quad k \in \mathbf{Z}_{\varepsilon} \\ 1 & x \in \Omega \cap Y_{\varepsilon k}, \quad k \notin \mathbf{Z}_{\varepsilon} .\end{cases}
$$

The set $\Omega_{\varepsilon}$ is quasi-periodic in the sense that it is divided in periodic cells, each one containing a hole which changes from cell to cell.

A law has to be given which says how the holes move:

- for a fixed $\varepsilon$, from one cell to another,

- when $\varepsilon$ moves.

We call this a microstructure. Let us define it mathematically. We do it in a way which fits the optimization techniques we have in mind.

Let $T_{0}^{*} \subset Y$ be a reference hole, we define $Y_{0}^{*}=Y \backslash T_{0}^{*}$ which is a Lipschitz open set. Let $\Phi_{0} \subset W^{1, \infty}\left(Y, \mathbf{R}^{2}\right)$ be the set of all bilipschitzian homeomorphisms of $Y$ into $Y$, that coincide with the identity on the boundary of $Y$. It can be proved, see [4], that $\Phi_{0}$ is an open subset of the affine subspace $I+W_{0}^{1, \infty}\left(Y, \mathbf{R}^{2}\right)$. We define a microstructure as an element:

$$
B \in \mathbf{B}=\mathbf{C}^{1}\left(\bar{\Omega} ; \Phi_{0}\right) .
$$

For each $x \in \Omega$, we define $Y^{*}(x)=B(x)\left(Y_{0}^{*}\right)$ and deduce $\Omega_{\varepsilon}$ from equation (1) using the procedure described above.

In each cell $Y_{\varepsilon k}$ such that $k \in \mathbf{Z}_{\varepsilon}$, the complement of the hole has the following properties. Defining

$$
Y_{\varepsilon k, 1}^{*}=\frac{1}{\varepsilon} Y_{\varepsilon k}-k,
$$

we have

$$
Y_{\varepsilon k, 1}^{*}=\left\{y \in Y ; \exists z \in Y_{0}^{*}, y=B(\varepsilon y+\varepsilon k)(z)\right\} .
$$

We can prove ([4]) that $Y_{\varepsilon k, 1}^{*}=F_{\varepsilon, k}\left(Y_{0}^{*}\right)$ for some $F_{\varepsilon k} \in \Phi_{\mathbf{0}}$ with

$$
\left\|F_{\varepsilon k}-B(\varepsilon k)\right\|_{W^{1, \infty}} \rightarrow 0, \quad \text { when } \varepsilon \rightarrow 0 .
$$

Moreover, there exists $\varepsilon_{0}$ such that for $\varepsilon \leq \varepsilon_{0}$, all the sets $Y_{\varepsilon k, 1}^{*}$ are uniformly Lipschitz.

In this setting, the homogenization results obtained in [11] are valid. They give the following homogenized equations:

- the microscopic equations: For a given $x \in \Omega$, denoting $B(x)$ by $F$ and letting $Y^{*}(x)=B(x)\left(Y_{0}^{*}\right), \theta_{i}^{F}$ is the unique solution of the variational equation:

$$
\begin{aligned}
\theta_{i}^{F} \in H_{\sharp}^{1}\left(Y^{*}(x)\right), & & \int_{Y^{*}(x)} \theta_{i}^{F}(y) d y=0, \\
\forall \varphi \in H_{\sharp}^{1}\left(Y^{*}(x)\right), & & \int_{Y^{*}(x)} \varphi(y) d y=0,
\end{aligned}
$$




$$
\int_{Y^{*}(x)}<\nabla \theta_{i}^{F}, \nabla \varphi>(y) d y=-\int_{Y^{*}(x)}<\nabla \varphi, e_{i}>(y) d y .
$$

- the homogenized coefficients: From these functions the homogenized coefficients can be explicitely computed. For $i, j=1,2$ we have

$$
a_{i j}(F)=\delta_{i j} \int_{Y^{*}(x)} d y+\int_{Y^{*}(x)}<\nabla \theta_{i}^{F}, e_{j}>(y) d y .
$$

As $F$ still denotes $B(x)$, this defines a matrix $A(B(x))=\left(a_{i j}(B(x))\right)$ which is defined for each $x \in \Omega$. It can be proved that it is symmetric positive definite. In particular its determinant is never equal to zero.

- the macroscopic equation: The torsion function $\psi(B)$ is defined by

$$
\begin{aligned}
& \psi(B) \in H_{0}^{1}(\Omega), \\
& \int_{\Omega}<\frac{A(B(x))}{\operatorname{det} A(B(x))} \nabla \psi(B)(x), \nabla \phi(x)>d x=2 \int_{\Omega} \phi(x) d x, \forall \phi \in H_{0}^{1}(\Omega)
\end{aligned}
$$

\subsection{The optimization problem}

The function $\psi(B) \in H_{0}^{1}(\Omega)$ that has been found in the previous equation gives a measure of the mechanical resistance of the homogenized bar. We wish to choose the microstructure $B \in \mathbf{B}=\mathbf{C}^{1}\left(\bar{\Omega} ; \Phi_{0}\right)$ in order to make it as strong as possible. So we are interested in the problem:

$$
\max _{B \in \mathbf{B}} j(B) \quad \text { with } \quad j(B)=2 \int_{\Omega} \psi(B)(x) d x .
$$

Not much is known about existence and uniqueness in this kind of problem. As classically done, we give in the sequel a way to compute the differential of $j$ with respect to $B$, aiming at a numerical computation of a local optimum using a descent algorithm of gradient type. Constraints on $B$ should be treated. In this paper we limit ourselves to the computation of the differential of $j$ with respect to $B$, with mathematical proofs of differentiability.

We are facing an optimal control problem where the state $\psi(B)$ is the solution of equation (4). We can see that the design variable $B$ appears in the coefficients of equation (4), and in the same time in the spaces in which equations (2) are posed. So we will need results in classical optimal control governed by partial differential equations and also in optimal design. We recall some basic results in these fields.

\section{Optimal CONTROL RECALLS}

\subsection{A STANDARD RESULT IN OPTIMAL CONTROL}

Let $\mathbf{V}$ be a Hilbert space (state space), $\Lambda$ be a Banach space and $\Phi$ be an open subset of $\Lambda$ (control space). We are given functionals :

$$
\begin{aligned}
& a: \Phi \times \mathbf{V} \times \mathbf{V} \rightarrow \quad \mathbf{R} \\
& (\varphi, u, v) \mapsto a(\varphi ; u, v), \\
& L: \Phi \times \mathbf{V} \rightarrow \mathbf{R} \\
& (\varphi, v) \mapsto L(\varphi ; v), \\
& J: \Phi \times \mathbf{V} \rightarrow \mathbf{R} \\
& (\varphi, v) \mapsto J(\varphi ; v) \text {. }
\end{aligned}
$$


For each $\varphi, a$ is supposed to be bilinear, continuous, symmetric, coercive in $u$ and $v ; L$ is supposed to be linear and continuous in $v$. Both are supposed to be of class $\mathbf{C}^{1}$ with respect to $\varphi$ respectively in the spaces of continuous bilinear functionals and continuous linear functionals. As for $J$, it is supposed to be $\mathbf{C}^{1}$ with respect to the pair $(\varphi, v)$.

We consider $u^{\varphi} \in \mathbf{V}$ uniquely defined by the state equation

$$
a\left(\varphi ; u^{\varphi}, v\right)=L(\varphi ; v), \quad \forall v \in \mathbf{V},
$$

and we let $j(\varphi)=J\left(\varphi, u^{\varphi}\right)$. We have the following classical result (see for instance [5]).

Theorem 1. The functions $\varphi \mapsto u^{\varphi}$ from $\Phi$ into $\mathbf{V}$ and $\varphi \mapsto j(\varphi)$ from $\Phi$ into $\mathbf{R}$ are $\mathbf{C}^{1}$. Moreover, for any $\delta \varphi \in \Lambda$

$$
\frac{d j(\varphi)}{d \varphi} . \delta \varphi=\frac{\partial J}{\partial \varphi}\left(\varphi ; u^{\varphi}\right) . \delta \varphi-\frac{\partial a}{\partial \varphi}\left(\varphi ; u^{\varphi}, p^{\varphi}\right) . \delta \varphi+\frac{\partial L}{\partial \varphi}\left(\varphi ; p^{\varphi}\right) . \delta \varphi,
$$

where $p^{\varphi}$ is the adjoint state, unique solution of the adjoint equation

$$
p^{\varphi} \in \mathbf{V}, \quad a\left(\varphi ; w, p^{\varphi}\right)=\frac{\partial J}{\partial v}\left(\varphi ; u^{\varphi}\right) . w, \quad \forall w \in \mathbf{V}
$$

\subsection{Optimal Design Basic technique}

The problem here is close to the setting of the preceding section. It is still a problem of optimal control, but the control now is the domain on which the partial differential equation associated with the bilinear form $a$ is posed. So, we consider some family $\Pi$ of open subsets $Z$ of $\mathbf{R}^{n}$ and for each $Z$ :

- a Hilbert space $\mathrm{V}(Z)$ depending on the control variable $Z$, and

-

$$
\begin{aligned}
& a^{Z}: \mathrm{V}(Z) \times \mathrm{V}(Z) \rightarrow \mathbf{R} \\
& u \quad, \quad v \quad \mapsto a^{Z}(u, v), \\
& L^{Z}: \mathbf{V}(Z) \rightarrow \mathbf{R} \\
& v \mapsto L^{Z}(v) \text {, } \\
& J^{Z}: \mathbf{V}(Z) \rightarrow \mathbf{R} \\
& v \mapsto J^{Z}(v) .
\end{aligned}
$$

For each $Z, a^{Z}$ is supposed to be bilinear, continuous, symmetric and coercive on $\mathbf{V}(Z), L^{Z}$ is linear continuous, and $J^{Z}$ is $\mathbf{C}^{1}$. The equation

$$
u^{Z} \in \mathbf{V}(Z), \quad a^{Z}\left(u^{Z}, v\right)=L^{Z}(v), \quad \forall v \in \mathbf{V}(Z)
$$

has one and only one solution. Defining $j(Z)=J^{Z}\left(u^{Z}\right)$, we want to differentiate $j$ with respect to $Z$.

This needs first to be defined because the variable $Z$ does not belong to a vector space. We work here in the very classical following setting (cf [6], [7], [8], [9], [10], [12], etc...). A regular part $Z_{0} \in \Pi$ is given as well as an open part $\mathbf{F}$ of $W^{k, \infty}\left(Z_{0}, \mathbf{R}^{n}\right)$ consisting of homeomorphisms of $Z_{0}$ into $\mathbf{R}^{n}$ with regularity $W^{k, \infty}$. We define $\Pi$ by

$$
\Pi=\left\{Z=F\left(Z_{0}\right) ; F \in \mathbf{F}\right\} .
$$

The spaces $\mathbf{V}(Z)$ we have in mind are Sobolev spaces. We choose $k$ such that the change of variable

$$
z=F\left(z_{0}\right)
$$


induces a topological isomorphism from $\mathrm{V}\left(Z_{0}\right)$ onto $\mathrm{V}(Z)$. The design variable is now $F \in \mathbf{F} \subset W^{k, \infty}\left(Z_{0}, \mathbf{R}^{n}\right)$. It belongs to a vector space. We are back to the setting of the previous section. We use its results.

Let us add in this section very classical formulas which will be used several times in what follows. One often has to deal with the bilinear form

$$
a^{Z}(u, v)=\int_{Z}<\nabla u(z), \nabla v(z)>d z
$$

where $\nabla u$ denotes the gradient of $u$ with respect to $z$. When one makes the change of variable $z=F\left(z_{0}\right)$, the gradients of $\hat{u}=u \circ F$ and $\hat{v}=v \circ F$ have to be taken with respect to $z_{0}$ instead of $z$. After computation one gets

$$
\left(\nabla_{z} u\right)\left(F\left(z_{0}\right)\right)=\left[D\left(F^{-1}\right) \circ F\left(z_{0}\right)\right]^{t} \cdot \nabla_{z_{0}} \hat{u}\left(z_{0}\right)
$$

so that

$$
\begin{aligned}
& a^{Z}(u, v)=\hat{a}(F ; \hat{u}, \hat{v}) \\
& =\int_{Z_{0}}<\left[D\left(F^{-1}\right) \circ F\right]^{t} \cdot \nabla \hat{u},\left[D\left(F^{-1}\right) \circ F\right]^{t} \cdot \nabla \hat{v}>\left(z_{0}\right)\left|\operatorname{det}(D \varphi)\left(z_{0}\right)\right| d z_{0} .
\end{aligned}
$$

Using the results of the previous subsection, we will have to differentiate the bilinear functional $\hat{a}(F ; .,$.$) with respect to F$. In a gradient method, if $F_{n}$ is the iterate $n$ this has to be done at each point $F_{n}$. Using an appropriate change of variable, it is well-known that it is sufficient to differentiate at the point $F=I$ (identity of $\mathbf{R}^{n}$ ). We will only need the formulas given in the following lemmas.

LEMMA 2. Let $\mathbf{F}$ be an open subset of the set of bilipschitz homeomorphisms of $Z_{0} \subset \mathbf{R}^{n}$ on their image. The mapping

$$
\mathbf{G}: F \mapsto D\left(F^{-1}\right) \circ F: \mathbf{F} \rightarrow\left[L^{\infty}\left(Z_{0}\right)\right]^{n^{2}}
$$

is $\mathbf{C}^{1}$ and its differential at the point $F=I$ (where $I$ stands for the identity of $\mathbf{R}^{n}$ ) is

$$
\left[\frac{d}{d F} \mathbf{G}(I) \cdot V\right]\left(z_{0}\right)=-D V\left(z_{0}\right), \quad \text { a.e. } z_{0} .
$$

LEMMA 3. In the same setting as in the previous lemma, let us consider the mapping

$$
\mathbf{H}: F \mapsto \operatorname{det} D F: \mathbf{F} \rightarrow L^{\infty}\left(Z_{0}\right) .
$$

It is $\mathbf{C}^{1}$ and its differential at the point $F=I$ is

$$
\left[\frac{d}{d F} \mathbf{H}(I) \cdot V\right]\left(z_{0}\right)=\operatorname{div} V\left(z_{0}\right), \quad \text { a.e. } z_{0} .
$$

\section{Differentiation of the functional $j$ With Respect to $B$}

We recall that we want to differentiate $j(B)=2 \int_{\Omega} \psi(B)(x) d x$ with respect to $B$, where $\psi(B)$ is defined by the sequence of equations (2), (3), (2.1). This can be done using the results of section 3 at different levels.

One is very simple: supposing that the coefficients of the matrix $A(B)$ are continuously differentiable with respect to $B$ from $\mathbf{B}$ into $L^{\infty}(\Omega)$, as $\operatorname{det} A(B)$ is not zero, we know from theorem 1 that $j$ is differentiable and from this same theorem, we have an explicit formula for its differential in terms of the differential of $A(B)$. More precisely, let us denote

$$
\tilde{a}_{i j}(B)(x)=a_{i j}(B(x)), \quad \tilde{A}(B)(x)=A(B(x)) .
$$

If we can prove that the functions $\tilde{a}_{i j}: \mathbf{B} \subset \mathbf{C}^{1}\left(\bar{\Omega} ; W^{1, \infty}\left(Y, \mathbf{R}^{2}\right)\right) \rightarrow L^{\infty}(\Omega)$ are $\mathbf{C}^{1}$ and if we have an explicit expression of their differential, we get the 
differential of $j$ directly from theorem 1 . So we first focalize on these $\tilde{a}_{i j}$ before coming back to $j(B)$.

\subsection{Differentiation of the homogenized coefficients}

We recall that they are defined by equations (2) and (3). Once again the differentiation of each $\tilde{a}_{i j}$ fits in the setting of section 3.2. Equation (2) is a state equation, and $\tilde{a}_{i j}$ is the functional that has to be differentiated. The results of section 3.1 are not sufficient because the state equation is posed on a space of functions which moves when $B$ does. That is why we have to use optimal design techniques.

First, we can simplify the problem with the help of the following lemma LEMMA 4. Recalling that $\tilde{a}_{i j}(B)(x)=a_{i j}(B(x))$ with $F=B(x) \in \Phi_{\mathbf{0}}$, supposing that the mapping $a_{i j}: \Phi_{\mathbf{0}} \subset W^{1, \infty}\left(Y, \mathbf{R}^{2}\right) \rightarrow \mathbf{R}$ is $\mathbf{C}^{1}$, then the mapping $\tilde{a}_{i j}: \mathbf{B} \subset \mathbf{C}^{1}\left(\bar{\Omega} ; W^{1, \infty}\left(Y, \mathbf{R}^{2}\right)\right) \rightarrow \mathbf{C}(\bar{\Omega})$ is also $\mathbf{C}^{1}$ and

$\forall H \in \mathbf{C}^{1}\left(\bar{\Omega} ; W_{0}^{1, \infty}\left(Y, \mathbf{R}^{2}\right)\right), \quad\left[\frac{d}{d B} \tilde{a}_{i j}(B) . H\right](x)=\frac{d}{d F} a_{i j}(B(x)) . H(x)$

(Notice that the perturbation $H$ has to be 0 on the boundary of $Y$ because $B+H$ must still be the identity on this boundary).

So, we can focalize on the simplified problem where $F \in \Phi_{0} \subset W^{1, \infty}\left(Y, \mathbf{R}^{2}\right)$ is the design variable instead of $B \in \mathrm{B}$. We let $Y^{*}=F\left(Y_{0}^{*}\right)$.

The first thing to do is to perform the change of variable $y=F\left(y_{0}\right)$ so that equation (2) is now posed on a fixed domain. We have to be careful that the zero-average condition does not work for this. Because of the change of variable $F$ appears, which is irrelevant. This can be by-passed by replacing the zero-average condition by the use of the quotient space $\mathbf{V}\left(Y^{*}\right)=H_{\sharp}^{1}\left(Y^{*}\right) / \mathbf{R}$, which by change of variable becomes $\mathbf{V}_{0}=H_{\sharp}^{1}\left(Y_{0}^{*}\right) / \mathbf{R}$. The state equation becomes (still denoting $\theta_{i}^{F}$ for its equivalence class)

$$
\begin{gathered}
\theta_{i}^{F} \in \mathbf{V}\left(Y^{*}\right), \\
\int_{Y^{*}}<\nabla \theta_{i}^{F}, \nabla \varphi>(y) d y=-\int_{Y^{*}}<\nabla \varphi, e_{i}>(y) d y, \forall \varphi \in \mathbf{V}\left(Y^{*}\right) .
\end{gathered}
$$

Now we fit exactly section 3.2. We get (see [4] for more details):

Theorem 5. Let $F \in \Phi_{\mathbf{0}}$ and $H \in W_{0}^{1, \infty}\left(Y, \mathbf{R}^{2}\right)$ be given. We define $V \in W_{0}^{1, \infty}\left(Y, \mathbf{R}^{2}\right) b y$

$$
I+V=(F+H) \circ F^{-1},
$$

(so that $\left.(I+V)\left(Y^{*}\right)=(F+H)\left(Y_{0}^{*}\right)\right)$.

The adjoint state is given by:

$$
\begin{gathered}
p_{i}^{F} \in \mathbf{V}\left(Y^{*}\right), \\
\int_{Y^{*}}<\nabla p_{i}^{F}, \nabla \varphi>(y) d y=-\int_{Y^{*}}<\nabla \varphi, e_{j}>(y) d y, \forall \varphi \in \mathbf{V}\left(Y^{*}\right),
\end{gathered}
$$

(so that $p_{i}^{F}=-\theta_{j}^{F}$ ), and

$$
\begin{gathered}
\frac{d}{d F} a_{i j}(F) \cdot H= \\
\delta_{i j} \int_{Y^{*}} \operatorname{div} V(y) d y+\int_{Y^{*}}<\nabla \theta_{i}^{F}(y), \nabla \theta_{j}^{F}(y)>\operatorname{div} V(y) d y \\
-\int_{Y^{*}}\left[<\nabla \theta_{i}^{F}(y), D V(y) \cdot e_{j}>+<\nabla \theta_{j}^{F}(y), D V(y) \cdot e_{i}>\right] d y
\end{gathered}
$$


OPTIMIZATION OF NON PERIODIC HOMOGENIZED MICROSTRUCTURES 171

$$
\begin{gathered}
-\int_{Y^{*}}\left[<\nabla \theta_{i}^{F}(y), e_{j}>+<\nabla \theta_{j}^{F}(y), e_{i}>\right] \operatorname{div} V(y) d y \\
-\int_{Y^{*}}<\left(D V+(D V)^{t}\right) \cdot \nabla \theta_{i}^{F}(y), \nabla \theta_{j}^{F}(y)>d y .
\end{gathered}
$$

Notice that all the functions involved in this formula either are given, or are computed through a coercive partial differential equation. So if $H$ is chosen in a finite dimensional vector space (using spline functions for instance), $\frac{d}{d F} a_{i j}(F) . H$ can be approximately computed using finite elements for instance.

Now we know the differential of the homogenized coefficients, we can go back to the macroscopic problem.

\subsection{Differentiation of the macroscopic problem}

This is a direct application of section 3.1. First we have (see [4]): Proposition 6. Let $\Gamma$ be defined by

$$
\Gamma(F)=\frac{A(F)}{\operatorname{det}(A(F)}
$$

(where $A(F)$ is the $2 \times 2$ matrix with coefficients $a_{i j}(F)$ ). This mapping $\Gamma: \Phi_{0} \subset W^{1, \infty}\left(Y, \mathbf{R}^{2}\right) \rightarrow\left[L^{\infty}(\Omega)\right]^{4}$ is $\mathbf{C}^{1}$ and for all $H \in W_{0}^{1, \infty}\left(Y, \mathbf{R}^{2}\right)$

$$
\frac{d}{d F} \Gamma(F) . H=[\operatorname{det} A(F)]^{-1}\left\{\frac{d}{d F} A(F) . H-\operatorname{tr}\left[A^{-1}(F) \frac{d}{d F} A(F) . H\right] A\right\} .
$$

Corollary 7 . For $\left.B \in \mathbf{B} \subset \mathbf{C}^{1}\left(\bar{\Omega} ; \Phi_{0}\right)\right)$, let $\tilde{\Gamma}(B)$ be defined by $\tilde{\Gamma}(B)=\Gamma(B(x))$. For any $\delta B \in \mathbf{C}^{1}\left(\bar{\Omega} ; W_{0}^{1, \infty}\left(Y, \mathbf{R}^{2}\right)\right)$ we have

$$
\left[\frac{d}{d B} \tilde{\Gamma}(B) . \delta B\right](x)=\frac{d}{d F} \Gamma(B(x)) . \delta B(x) .
$$

Notice that theorem 5 , proposition 6 , corollary 7 used sequentially, give the differential of $\tilde{\Gamma}$ in the direction $\delta B$. In theorem $5, H$ has to be taken equal to $\delta B(x)$.

Now, using again section 3.1, we get:

ThEOREM 8. The functional $j(B)=\int_{\Omega} \psi(B)(x) d x$, where $\psi(B)$ is defined by equation (2.1), is $\mathbf{C}^{1}$. The adjoint state $p(B)$ is equal to $\psi(B)$. We have

$$
\frac{d}{d B} j(B) . \delta B=-\int_{\Omega}<\left[\frac{d}{d B} \tilde{\Gamma}(B) \cdot \delta B\right] \cdot \psi(B), \psi(B)>(x) d x
$$

where $\frac{d}{d B} \tilde{\Gamma}(B) . \delta B$ is given in corollary $\gamma$.

This finally gives the differential of the given functional $j(B)$.

\section{Numerical remark:}

This differential can be computed with the sequence of formulas given in theorem 5, proposition 6 , corollary 7 and theorem 8 . Nobody would like to develop these formulas explicitly. This sequence can be directly written in a programming language (FORTRAN could do the job if nothing more sophisticated is available). They better be written as independent subroutines, one eventually calling the previous ones for adapted values of the input parameters. 


\section{REFERENCES}

[1] Kikuchi N., Bendsoe M., Generating optimal topologies in optimal design using an homogemization method, Computer methods in applied mechanics and engineering, vol.71, pp.197-224, 1988

[2] Kohn R.V., Strang G., Optimal design and relaxation of variational problems, Communications in pure and applied mathematics, vol.39, pp.1-25, pp.139-182, pp.353377,1986

[3] Kohn R.V., Strang G., Optimal design in elasticity and plasticity, Int. J. Numer. Methods and Engrg., vol.22, pp. 183-188, 1986

[4] Chenais D., Mascarenhas M.L., Trabucho L., On optimization of non-periodic homogenized microstructures, Applied Math. and Optimization, to be published

[5] Chenais D., Optimal design of midsurface of shells: differentiability proof and sensitivity computation, Applied Math. and Optimization, vol. 16, pp. 93-133, 1987

[6] Rousselet B., Optimization of distributed parameter structures, NATO Advanced Study Institutes series, 50, Sijthoff and Noordhoff, 1981

[7] Zolesio J.P., Optimization of distributed parameter structures, NATO Advanced Study Institutes series, 50, Sijthoff and Noordhoff, 1981

[8] Murat F., Simon J., Sur le contrôle par un domaine géométrique, Publications du Laboratoire d'Analyse Numérique, Université Pierre et Marie Curie, Paris, 1976

[9] Guillaume P., Dérivées d'ordre supérieur en optimisation de forme, Thèse de l'Université de Toulouse, France, 1994

[10] Masmoudi M., Outils pour la conception optimale de forme, Thèse de l'Université de Nice, France, 1987

[11] Mascarenhas M.L., Polisevski D., The warping, the torsion and the Neumann problems in a quasi-periodically perforated domain, Mathematical Modeling and Numerical Analysis, vol.28 (1), pp.37-57, 1994

[12] Chenais D., Optimisation de domaines, Cours de troisième cycle, 1992

D. Chenais: Laboratoire de mathématiques, Université de NiCE, BP 71, 06108 Nice Cedex, France. M.L. Mascarenhas, L.Trabucho: Centro de matematica, Universidade de LisboA, Av. Prof. Gama Pinto 2, 1699 Lisboa Codex, Portugal 